\title{
Kinetic Explanations for the Sequence Biases Observed in the Nonenzymatic Copying of RNA Templates
}

\author{
Dian Ding ${ }^{1,2,4}$, Lijun Zhou ${ }^{1,3,4}$, Constantin Giurgiu' ${ }^{1,2}$ and Jack W. Szostak ${ }^{1,2,3}$ *
}

${ }^{1}$ Howard Hughes Medical Institute, Department of Molecular Biology and Center for Computational and Integrative Biology, Massachusetts General Hospital, 185 Cambridge Street, Boston, Massachusetts 02114, USA.

${ }^{2}$ Department of Chemistry and Chemical Biology, Harvard University, 12 Oxford Street, Cambridge, Massachusetts 02138, USA.

${ }^{3}$ Department of Genetics, Harvard Medical School, 77 Avenue Louis Pasteur, Boston, Massachusetts 02115, USA.

${ }^{4}$ These authors contributed equally to this work.

${ }^{*}$ To whom correspondence should be addressed. Tel: +1 617726 5102; Fax: +1 617643 3328; Email: szostak@molbio.mgh.harvard.edu

Present Address: Constantin Giurgiu, Carl R. Woese Institute for Genomic Biology, 1206 W Gregory Dr, Urbana, Illinois 61801, USA. 


\begin{abstract}
The identification of nonenzymatic pathways for nucleic acid replication is a key challenge in understanding the origin of life. We have previously shown that nonenzymatic RNA primer extension using 2-aminoimidazole (2Al) activated nucleotides occurs primarily through an imidazolium-bridged dinucleotide intermediate. The reactive nature and preorganized structure of the intermediate increase the efficiency of primer extension but remain insufficient to drive extensive copying of RNA templates containing all four canonical nucleotides. To understand the factors that limit RNA copying, we synthesized all ten 2Al-bridged dinucleotide intermediates and measured the kinetics of primer extension in a model. The affinities of the ten dinucleotides for the primer/template/helper complexes vary by over 7,000 -fold, consistent with nearest neighbor energetic predictions. Surprisingly, the reaction rates at saturating intermediate concentrations still vary by over 15 -fold, with the most weakly binding dinucleotides exhibiting a lower maximal reaction rate. Certain noncanonical nucleotides can decrease sequence dependent differences in affinity and primer extension rate, while monomers bridged to short oligonucleotides exhibit enhanced binding and reaction rates. We suggest that more uniform binding and reactivity of imidazolium-bridged intermediates may lead to the ability to copy arbitrary template sequences under prebiotically plausible conditions.
\end{abstract}




\section{INTRODUCTION}

The absence of any known pathway for the efficient nonenzymatic copying of RNA templates that contain all four nucleotides is one of the main obstacles towards understanding the emergence of the first ribozymes and hence the origin of primordial RNA based life. Leslie Orgel pioneered the study of this subject by showing that imidazole and 2-methyl-imidazole activated 5'-monophosphate ribonucleotides could polymerize on RNA templates.(1-3) Recently, the mechanism of nonenzymatic primer extension mediated by 2-methyl-imidazolium-5'-phospho-ribonucleotides has been unraveled.(4) Surprisingly, the dominant reaction mechanism is not a simple $S_{N} 2$ reaction in which the 3'-OH of the primer attacks the 5'phosphate of an adjacent template-bound activated mononucleotide. Instead, two activated monomers first react with each other to form a covalent intermediate in which the two mononucleotides flank a central imidazolium group (Figure 1A). The intermediate dinucleotide binds to the template at the +1 and +2 positions via Watson-Crick base pairs, after which the 3'-hydroxyl group of the primer attacks the proximal phosphate group, resulting in a +1 -extension product. The nucleotide bound at the +2 position of the template, together with the imidazolium-phosphate moiety, acts as the leaving group in this reaction. Primer extension via direct attack on an activated monomer can occasionally occur but this reaction is far slower than the reaction with an imidazolium-bridged dinucleotide.(5) Sequencing of the products of primer extension has also shown that monomer-mediated primer extension is highly error prone compared to primer extension with imidazolium-bridged dinucleotides. $(6,7)$

Nucleotide activation with 2-aminoimidazole leads to increased rates and yields of primer extension compared to 2-methylimidazole or imidazole.(8) The superior properties of 2-aminoimidazole are attributable at least in part to the greater stability of the corresponding bridged dinucleotide intermediates, which accumulate to higher levels resulting in more efficient primer extension. Moreover, a potentially prebiotic pathway for the synthesis of 2-aminoimidazole shares a common mechanism with and can occur in the same reaction mixture as that of 2-aminooxazole, a precursor of prebiotic nucleotide synthesis(9). For these reasons we believe that 2-aminoimidazole activated ribonucleotides are the best available model for the study of nonenzymatic template-directed primer extension reactions.

Despite the enhanced rate and fidelity of primer extension via imidazolium-bridged dinucleotides, this process is only efficient on $G+C$ templates, while templates containing $A, G, C$ and $U$ are copied very poorly. Although some bias against $A$ and $U$ is to be expected given the weaker $A: U$ base-pair compared to the $\mathrm{G}: \mathrm{C}$ base-pair, a deeper mechanistic understanding of the template copying process is required to solve the problem of copying mixed sequence templates. Until now, a detailed kinetic study of primer extension with 2-amino-imidazolium-bridged dinucleotides has only been done with the dicytidine intermediate $\left(C^{*} C\right) .(10)$ This study provided a kinetic framework for the synthesis and breakdown of the intermediate, as well as the greater reactivity of the dinucleotide intermediate relative to the activated monomer. However, since only $C^{*} C$ was studied, this study did not address the problem of copying arbitrary RNA sequences. Recently, our lab has reported the use of deep sequencing to study nonenzymatic RNA primer extension on random sequence templates(7). As expected, we observed an 
initial bias favoring primer extension with $\mathrm{G}$ and $\mathrm{C}$, but surprisingly continued primer extension became even more strongly biased in favor of $\mathrm{C}$. Template sequencing suggested that this bias resulted from the superior activity of the $\mathrm{C}^{*} \mathrm{C}$ dinucleotide relative to all other $\mathrm{N}^{*} \mathrm{~N}$ dinucleotide intermediates. In order to understand the kinetic basis for this result, kinetic studies of the other $\mathrm{N}^{*} \mathrm{~N}$ intermediates are needed. Here we report the chemical synthesis and characterization of all ten dinucleotide intermediates $\left(A^{*} A\right.$, $C^{*} C, G^{*} G, U^{*} U, A^{*} C, A^{*} G, A^{*} U, C^{*} G, C^{*} U$ and $G^{*} U$ ) and the concentration dependent reaction rates of each dinucleotide when bound to its respective Watson-Crick base pairing template. Our results reveal a surprisingly broad range of affinities and reaction rates. Intriguingly, the identity of the downstream nucleotide significantly affects the rate of primer extension. NMR studies of sugar pucker conformation provide a potential explanation for the preferential incorporation of $\mathrm{C}$ during RNA copying. Preliminary kinetic studies of bridged dinucleotides containing the noncanonical nucleotide 2-thio- $U$, as well as monomers bridged to short oligonucleotides, suggest strategies for the further optimization of the nonenzymatic copying of arbitrary RNA sequences.

\section{MATERIAL AND METHODS}

\section{Synthesis and characterization of 2-aminoimidazolium-bridged dinucleotide intermediates}

The homo-dimers $A^{*} A, C^{*} C$ and $U^{*} U$ were synthesized by mixing 1 equivalent of nucleoside $5^{\prime}$ monophosphate with 0.45 equivalent of 2 -aminoimidazole (2Al) in dry dimethyl sulfoxide (DMSO), then adding 20 equivalents of triethylamine (TEA), 10 equivalents of triphenylphosphine (TPP) and 10 equivalents of 2,2'-dipyridyldisulfide (DPDS). After incubation for 30 mins, the product was precipitated in acetone with sodium perchlorate. The precipitant was washed twice by mixture of acetone and diethyl ether $(1: 1, v / v)$ and dried under vacuum. Then the dry pellet was resuspended in $20 \mathrm{mM}$ triethylaminebicarbonate buffer (TEAB), pH 8.0, and purified by reverse-phase flash chromatography, with a $50 \mathrm{~g}$ C18Aq column. The desired products were separated from other compounds by reverse phase flash chromatography over $12 \mathrm{CVs}$ of $0-10 \%$ acetonitrile in $2 \mathrm{mM}$ TEAB buffer ( $\mathrm{pH} 8.0$ ). We did not use this method for $G^{*} G$, because the low solubility issue of guanosine $5^{\prime}$-monophosphate (GMP) prevented us from mixing GMP with $2 \mathrm{Al}$ at the desired ratio.

To synthesize $G^{*} G$ and the six hetero-dimers $\left(N^{*} N^{\prime}\right)$, we first synthesized and purified $2 A$ l activated $N$ monomer and 1-hydroxy-7-azabenzotriazole (HOAt) activated $\mathrm{N}^{\prime}$ monomer, as described above except that 10 equivalents of $2 \mathrm{Al}$ or HOAt were added. To overcome the poor solubility of GMP in DMSO, GMP was first dissolved in water together with 10 equivalents of $2 \mathrm{Al}$ or HOAt and $100 \mathrm{mM}$ TEA, flash frozen and lyophilized, and then dissolved in DMSO for further reactions. Then purified 2Al-activated $\mathrm{N}$ and HOAt-activated $\mathrm{N}^{\prime}$ were mixed in a 1:1 molar ratio in water, at $\mathrm{pH} 8.0$ (adjusted by $\mathrm{NaOH} / \mathrm{HCl}$ ). $\mathrm{HOAt}$ is a good leaving group but cannot form a 5'-5' bridged intermediate like 2AI. After incubation for 30-60 minutes, the $2 \mathrm{Al}$ intermediate dimer $\mathrm{N}^{*} \mathrm{~N}^{\prime}$ was the predominant product, alongside byproducts such as $\mathrm{N}^{*} \mathrm{~N}, \mathrm{~N}^{\prime *} \mathrm{~N}^{\prime}$, and activated monomers. The desired product was then purified by preparative scale HPLC using a C18 reverse phase column and a solvent gradient (A) $2 \mathrm{mM}$ aqueous TEAB buffer, pH 8.0, (B) 
acetonitrile. The gradients for each different bridged dinucleotide are described in the supplementary information.

The synthesis of the noncanonical bridged dinucleotides $\mathrm{SU}^{*} \mathrm{sU}$ and $\mathrm{SC} \mathrm{SC}^{*}$ started with phosphorylation of 2-thiouridine and 2-thiocytidine to form the 5'-monophosphate sUMP and SCMP. 1 equivalent of the 5'hydroxyl nucleotide was dissolved in an ice-chilled solution of 80 equivalents of trimethyl phosphate, 3.6 equivalents of phosphoryl chloride, and 0.4 equivalent of water. Then four separate portions of $N, N$ diisopropylethylamine ( 0.52 equiv. each) were added dropwise to the stirred solution with 20 min intervals at $0^{\circ} \mathrm{C}$. The reaction mixture was stirred for another 20 min and quenched with $1 \mathrm{M} \mathrm{TEAB}, \mathrm{pH} 7.6$. The mixture was purified first by reverse phase flash chromatography over $5 \mathrm{CVs}$ using $25 \mathrm{mM}$ TEAB buffer $(\mathrm{pH}$ 7.5). Fractions containing product were lyophilized and the resultant syrup was purified again by reverse phase chromatography over $10 \mathrm{CVs}$ of $0-10 \%$ acetonitrile in $25 \mathrm{mM}$ TEAB buffer $(\mathrm{pH} 7.5)$ to give sUMP or SCMP. The activation of sUMP and SCMP using $2 \mathrm{Al}$ led to a low yield $(10 \sim 20 \%)$ and we were unable to synthesize HOAt-activated $\mathrm{sU}$ or $\mathrm{sC}$. Therefore, $\mathrm{SU}^{*} \mathrm{sU}$ and $\mathrm{SC}^{*} \mathrm{sC}$ were synthesized by activating the nucleotide monophosphate using 2Al-activated monomer as follows. SUMP or sCMP was activated using $2 \mathrm{Al}$ as described above to form $2 \mathrm{Al}-\mathrm{sU}$ or $2 \mathrm{Al}-\mathrm{sC}$. Then 1 equivalent of $2 \mathrm{Al}-\mathrm{sU}$ or $2 \mathrm{Al}-\mathrm{sC}$ and 1.15 equivalent of sUMP or SCMP were incubated with 30 equivalents of TPP and 30 equivalent of DPDS for $2 \mathrm{~h}$ in DMSO using a similar activation procedure. The desired product was then purified by reverse phase flash chromatography over $25 \mathrm{CVs}$ of $0-10 \%$ acetonitrile in $2 \mathrm{mM}$ TEAB buffer $(\mathrm{pH} 7.5)$. Monomer-bridged-oligonucleotides were made from 2Al activated oligonucleotides and HOAt-activatedpA. The 5'-phosphorylated oligonucleotides ( $\mathrm{pCG}, \mathrm{pCGC}$, and pCGCA) were prepared by solid-phase RNA synthesis. The oligonucleotide 5'-monophosphate was then activated using 40 equivalents of $2 \mathrm{Al}$, 40 equivalents of TPP, and 40 equivalents of DPDS in DMSO and TEA for 6 hours following a similar activation procedure as described above. The 2Al-activated-oligonucleotide was then mixed with 1.5 equivalent of HOAt-activated-pA in water at $\mathrm{pH} 8.0$ for $1 \mathrm{~h}$. The mixture was purified by preparative scale HPLC using a C18 reverse phase column over $27 \mathrm{CVs}$ of $0-10 \%$ acetonitrile in $25 \mathrm{mM}$ TEAB buffer $(\mathrm{pH}$ 7.5).

The detailed synthesis procedure for each individual bridged intermediate as well as their characterizations are included in the supplementary data.

\section{Nonenzymatic primer extension reactions}

Primer/template/helper duplexes were prepared in an annealing buffer at $5 x$ the final concentration. Solutions containing $7.5 \mu \mathrm{M}$ primer, $12.5 \mu \mathrm{M}$ template, $17.5 \mu \mathrm{M}$ helper, $50 \mathrm{mM}$ Tris- $\mathrm{Cl}$ ( $\mathrm{pH}$ 8.0), $50 \mathrm{mM}$ $\mathrm{NaCl}$ and $1 \mathrm{mM}$ EDTA ( $\mathrm{pH} \mathrm{8.0)}$ were heated at $95^{\circ} \mathrm{C}$ for $30 \mathrm{~s}$ and then slowly cooled to $25^{\circ} \mathrm{C}$ at a rate of $0.1^{\circ} \mathrm{C} / \mathrm{s}$ in a thermal cycler machine. The annealed products were then diluted five-fold into the primer extension reaction buffer containing $200 \mathrm{mM}$ Tris- $\mathrm{Cl}$, pH 8.0, and $100 \mathrm{mM} \mathrm{MgCl}_{2}$. Stock solutions of imidazolium-bridged dinucleotide intermediates were freshly prepared and added to the buffer with annealed primer/template/helper duplexes to initiate primer extension reactions. At each time point, 0.5 
$\mu \mathrm{L}$ of reaction sample was added to $25 \mu \mathrm{L}$ quenching buffer containing $20 \mathrm{mM}$ EDTA, 1x TBE and $10 \mu \mathrm{M}$ of an RNA complementary to the template.

\section{Urea-PAGE analysis}

Primer extension products were resolved by 20\% (19:1) polyacrylamide gel electrophoresis (PAGE) with $7 \mathrm{M}$ urea, in 1x TBE gel running buffer. The gels were scanned with an Amersham Typhoon RGB Biomolecular Imager (GE Healthcare Life Sciences). The fluorescently-labeled primer and extended primer bands were visualized, and then quantified using ImageQuant TL software to obtain relative band intensities.

\section{NMR measurement of sugar conformations}

Proton NMR spectra of all imidazolium-bridged dinucleotides were acquired on a Varian Oxford AS-400 NMR spectrometer (for details see the Supplementary Information). Proton NMR peak assignments were performed with the help of coupling constants and 2D COSY NMR. For all the hetero-dinucleotide intermediates, the two different nucleotides in the molecule have well separated $\mathrm{H} 1$ ' hydrogen peaks. The ${ }^{3} \mathrm{JH}_{1}{ }^{\prime}-\mathrm{H}^{\prime}$ coupling constant was measured from the NMR peak corresponding to $\mathrm{H} 1$ ' only, because the $\mathrm{H} 2$ ' peak overlaps with other peaks for most of the bridged dinucleotides. The ratio of the fraction of 3'endo to 2'-endo sugar pucker conformation was calculated based on the equation: C3'-endo/2'-endo = $\mathrm{J} /(10-\mathrm{J})$ where $\mathrm{J}={ }^{3} \mathrm{~J}_{\mathrm{H} 1}{ }^{\prime}-\mathrm{H} 2 \cdot$. $(11)$

\section{RESULTS}

\section{Stability of imidazolium-bridged dinucleotides}

To investigate the reasons for the strong sequence bias in nonenzymatic RNA copying, we conducted a comparative study of the kinetics of template copying using purified imidazolium-bridged dinucleotide intermediates. In order to avoid artifacts due to possible differences in chemical stability, we first measured the rates of hydrolysis of selected $\mathrm{N}^{*} \mathrm{~N}$ intermediates. The degradation rate of the homodinucleotide $C^{*} C$ has previously been reported as $0.17 \mathrm{~h}^{-1}$ (half-life $\sim 245 \mathrm{~min}$ )(10), under slightly different buffer conditions than used here (90 mM Tris ( $\mathrm{pH}$ 8.3-8.4) and $90 \mathrm{mM} \mathrm{MgCl}$ ). We used proton NMR to follow the degradation of $A^{*} C$ and $A^{*} U$, two representative hetero-dinucleotides, under our primer extension reaction conditions (200 mM Tris 8.0 and $100 \mathrm{mM} \mathrm{MgCl}_{2}$, Figure S1). We found that both $A^{*} \mathrm{C}$ and $A^{*} U$ degraded by hydrolysis to give $2 \mathrm{Al}$-activated monomers and unactivated 5 '-monophosphate monomers; these compounds subsequently reacted to form imidazolium-bridged homo-dinucleotides and other products. As a result, the overall degradation process did not follow simple pseudo-first-order reaction kinetics. Nevertheless, from the time-course curves, half-lives for $A^{*} C$ and $A^{*} U$ of $\sim 135$ min and $\sim 120$ min respectively were estimated (Figure S1). At $10 \mathrm{~min}, 95 \%$ and $94 \%$ of $A^{*} C$ and $A^{*} U$ respectively remained unhydrolyzed. We conclude that primer extension reaction rates obtained within ten minutes 
should be reasonably accurate and should allow for valid comparisons between different imidazoliumbridged dinucleotides.

\section{Nonenzymatic primer extension reactions with $5 \mathrm{mM}$ intermediate}

For an initial comparison of the reaction rates of the ten different $\mathrm{N}^{*} \mathrm{~N}$ intermediates, we set up ten different primer extension reactions, each with $5 \mathrm{mM}$ of one intermediate and its complementary template. The experimental design is illustrated in Figure 1C, with the 26-nt template, 12-nt primer and 12-nt downstream helper $\left(5^{\prime}-\mathrm{OH}\right)$. Each intermediate $\mathrm{N} 1{ }^{*} \mathrm{~N} 2$ binds to the template through two Watson-Crick base-pairs with the nucleotides N3 and N4, and is sandwiched between the 3'-guanosine of the primer and the 5'-guanosine of the downstream helper oligonucleotide. Without the stabilizing effect of the stacking interaction with the downstream helper, the reactions of some intermediates (e.g., $U^{*} U$ ) are barely detectable. This experimental design allowed us to make a direct comparison of the pseudo-firstorder reaction rates $\left(k_{\text {obs }}\right)$ of all ten intermediates in sixteen parallel sets of reactions, as shown in Figure 2.

At a concentration of $5 \mathrm{mM}$ for each imidazolium-bridged dinucleotide, the observed rates of primer extension varied over a range of 240 -fold, with the lowest $k_{\text {obs }}$ of $0.35 \mathrm{~h}^{-1}$ for $\mathrm{U}^{*} \mathrm{U}$ and the highest $k_{\text {obs }}$ being $84 \mathrm{~h}^{-1}$ for $\mathrm{C}^{*} \mathrm{G}$. The reaction rates in Figure 2 are consistent with previous studies of 2methylimidazole activated mono-nucleotides, which form analogous imidazolium-bridged homodinucleotide intermediates, in that primer extension with $G^{*} G$ and $C^{*} C$ are the most rapid, while primer extension with $A^{*} A$ is slower(4). However, when we examined the reaction rates of the heterodinucleotide intermediates, we found surprising differences depending on the $5^{\prime}-3^{\prime}$ orientation of the intermediate on the template. For example, primer extension with $5^{\prime}-U^{*} G\left(41 h^{-1}\right)$ is four-fold faster than with $5^{\prime}-G^{*} U\left(10 \mathrm{~h}^{-1}\right)$ even though this intermediate has the same Watson-Crick base pairing in both cases. Similarly, primer extension with $5^{\prime}-U^{*} A\left(11 h^{-1}\right)$ is six-fold faster than with $5^{\prime}-A^{*} U\left(1.7 h^{-1}\right)$. Comparing the reaction rates in the columns of Figure 2 shows that the identity of the downstream base significantly affects the primer extension rate. With the same $\mathrm{N} 1$, the trend for kobs generally follows $\mathrm{N} 1{ }^{*} \mathrm{U}<\mathrm{N} 1{ }^{*} \mathrm{C} \leq$ $\mathrm{N} 1^{*} \mathbf{A}<\mathrm{N} 1^{*} \mathbf{G}$, consistent with a favorable influence of the stronger $\mathrm{C} \cdot \mathrm{G}$ vs. $A \cdot U$ base-pair, combined with the stronger stacking interaction of purines $(A, G)$ than pyrimidines $(C, U)$. For a given $N 2$, the trend of $k_{\text {obs }}$ is $\mathbf{U}^{*} \mathrm{~N} 2<\mathbf{A}^{*} \mathrm{~N} 2<\mathbf{G}^{*} \mathrm{~N} 2 \leq \mathbf{C}^{*} \mathrm{~N} 2$ in general, consistent with previous studies showing that primer extension rates with $C$ and $G$ are robust, following by $A$, while the extension of $U$ is very poor. However, when $\mathrm{N} 2=\mathrm{G}$, the trend of $\mathrm{N} 1$ reaction rate is $\mathrm{U}<\mathrm{G}<\mathrm{A}<\mathrm{C}$. Although the extension rates of all four nucleobases are rapid, this different order was surprising, and led us to ask whether the different observed rates reflected differences in binding or reactivity for the different intermediate/template combinations.

\section{Kinetic study of nonenzymatic primer extension reactions}


We sought to determine whether the differences in the observed primer extension reaction rates were due to differences in the affinities or the reactivities of the imidazolium-bridged dinucleotide intermediates. To answer this question, we measured the rates of primer extension as a function of the concentration of each intermediate. This allowed us to obtain the observed maximal rate ( $\left.k_{\text {obs } \max }\right)$, when the template is saturated with the intermediate (Figure 3). We also fitted the data to Michaelis-Menten equation and generated values for $\mathrm{K}_{\mathrm{m}}$, which reflects the affinity of each dimer (Figure 4, S2). Though MichaelisMenten models are used for enzyme kinetic studies, they are also suitable for the case of nonenzymatic primer extension reactions. Firstly, the concentrations of imidazolium-bridged dinucleotides ("substrates") are three orders of magnitudes higher than the concentrations of primer/template/helper duplexes ("enzymes"). Secondly, the reactions are also two step reactions, in which a rapid reversible step of dinucleotide binding is followed by an irreversible step of phosphodiester bond formation catalyzed by the primer/template/helper complex. As is the case with enzymatic reactions, $\mathrm{K}_{\mathrm{m}}$ is not necessarily equal to $\mathrm{K}_{\mathrm{d}}$, but is a close approximation of $\mathrm{K}_{\mathrm{d}}$ as long as the on and off rates of the 'substrate' imidazoliumbridged dinucleotide are fast relative to the rate of the chemical step. Due to the reactive nature of the dinucleotide substrates, we have not yet been able to directly measure $K_{d}$ values by physical methods. To avoid the effect of differing affinities, we compared the reactivity of each dimer under conditions of template saturation ( $\left.k_{\text {obs max }}\right)$. The results showed a 15 -fold difference between the lowest $\left(5.7 \mathrm{~h}^{-1}\right.$ for $\left.\mathrm{U}^{*} \mathrm{U}\right)$ and the highest reaction rate $\left(85 \mathrm{~h}^{-1}\right.$ for $\left.C^{*} G\right)$. Under saturation conditions the variation in reaction rates is decreased relative to the scenario in which all intermediates were present at the same low concentration, consistent with significant differences in affinity contributing to the larger range of reaction rates when all intermediates were present at the same concentration. Surprisingly, however, the trends in reaction rates were similar at $5 \mathrm{mM}$ and at saturating concentrations. For a given $\mathrm{N} 1$, reaction rates followed $\mathrm{N} 1^{*} \mathrm{U}<$ $\mathrm{N} 1{ }^{*} \mathrm{~A}<\mathrm{N} 1{ }^{*} \mathrm{C}<\mathrm{N} 1{ }^{*} \mathrm{G}$ for kobs max. We conclude that different imidazolium-bridged dinucleotides have intrinsic differences in reactivity.

Next, we measured the affinities of each imidazolium-bridged dinucleotide and asked what factors affect template binding. Figure 4 shows that the highest $K_{m}$ is $54 \mathrm{mM}\left(U^{*} U\right)$, while the lowest $K_{m}$ is $0.0071 \mathrm{mM}$ $\left(G^{*} G\right)$; the difference is almost four orders of magnitude. For a given $N 1$, the trend is $N 1^{*} U>N 1^{*} C>$ $N 1{ }^{*} A>N 1 * G$. For the same $N 2, K_{m}$ the trend is $U^{*} N 2>A^{*} N 2>C^{*} N 2>G^{*} N 2$ in general. The $K_{m}$ of $G^{*} C$ is surprisingly high at $1.4 \mathrm{mM}$ (vs. $0.03 \mathrm{mM}$ for $C^{*} \mathrm{G}$ ), indicating relatively weak binding affinity. This large difference must reflect that importance of stacking interactions in stabilizing the binding of imidazoliumbridged dinucleotides. This is also reflected in the 14-fold difference in affinities between $G^{*} U\left(K_{m}=11\right.$ $\mathrm{mM})$ and $U^{*} \mathrm{G}\left(\mathrm{K}_{\mathrm{m}}=0.80 \mathrm{mM}\right)$. Considering all pyrimidine*purine intermediates, the affinity is always greater when the pyrimidine is upstream. That is, the $K_{m}$ of $U^{*} A$ is less than the $K_{m}$ of $A^{*} U$, the $K_{m}$ of $C^{*} A$ is less than the $K_{m}$ of $A^{*} C$, and the $K_{m}$ of $C^{*} G$ is less than the $K_{m}$ of $G^{*} C$. The differences vary from 8 to 47 fold. The effect of binding orientation is minor in the case of purine* purine and pyrimidine*pyrimidine imidazolium-bridged intermediates, e.g. the $K_{m}$ of $U^{*} C$ is only 1.2-fold higher than the $K_{m}$ of $C^{*} U$, and the $K_{m} G^{*} A$ is only 2 -fold higher than the $K_{m}$ of $A^{*} G$. These effects are likely due to the sum of the stacking 
interactions with the guanosines that flank the bound imidazolium-bridged dinucleotides, e.g. the stacking interactions $55^{\prime}-G N_{1}-3^{\prime}$ and $5^{\prime}-N_{2} G-3^{\prime}$. It has been previously reported that the $5^{\prime}-P u-P y-3^{\prime}$ orientation results in greater energy of stacking than the $5^{\prime}-P y-P u-3^{\prime}$ orientation.(12) As a result, when $\mathrm{N}_{1}$ is a pyrimidine and $\mathrm{N}_{2}$ is a purine, the sum of the stacking interactions is predicted to be stronger than for the reverse case.

As noted above there is a correlation between the reactivities and the affinities of the imidazolium-bridged dinucleotide intermediates. One possible explanation is that strong binding of the intermediate stabilizes the configuration that is optimal for nonenzymatic primer extension. For example there could be situations where one nucleotide of the dinucleotide intermediate is bound, but the other one is fluctuating between the bound and the unbound state. If $\mathrm{N} 1$ of the dinucleotide is only Watson-Crick paired to the template a fraction of the time that the dinucleotide is bound, the rate of the primer extension reaction would be correspondingly decreased. More detailed studies of the physical characteristics of the bound intermediates are required to further understand their behavior in primer extension.

\section{The effect of ribose configuration on the reaction}

The main exception to the trends in binding and reactivity discussed above is the anomalously high reactivity of the imidazolium-bridged dinucleotides in which $\mathrm{N} 1$ is $\mathrm{C}$. This observation is consistent with the results from deep sequencing of the products of nonenzymatic primer extension on random templates, in which primer extension is dominated by the incorporation of C.(6) This cannot be explained solely by affinity, since the binding of $G$ should generally be stronger. What then could account for the greater reactivity if imidazolium-bridged dinucleotides with $\mathrm{C}$ at the $\mathrm{N} 1$ position? We asked if the conformational preference of the ribofuranose rings of the bridged dinucleotides could potentially explain this anomalous reactivity. The furanose rings of ribonucleotides can pucker in two different ways: the $\mathrm{C} 3$ '-endo and the $\mathrm{C} 2$ '-endo conformations. Although ribonucleotides generally prefer to adopt the C3'-endo configuration, the two conformations are close in energy and can easily interconvert in solution. Therefore, the conformational behavior of most nucleotides can be modeled as a two-state equilibrium; this has been extensively characterized by NMR.(13) Previous studies suggest that the C3'endo conformation for the template and 3'-terminal primer position is optimal in primer extension reactions.(14-16)

Activated 5'-GMP has been shown to undergo a sugar pucker switch from predominantly C2'-endo in solution to C3'-endo upon binding to an RNA template.(17) In addition, crystallographic studies show that the $G^{*} G$ intermediate exhibits only the $C^{\prime}$ '-endo configuration when bound to the template downstream of a primer.(15)

To understand the factors that influence the rates of nonenzymatic primer extension reactions, we determined the sugar pucker conformation of each ribonucleotide at the N1 position of all 10 imidazoliumbridged dinucleotides. We determined the ratio between the C3'- and C2'-endo conformations by NMR, making use of the well-established correlation between the ${ }^{3} \mathrm{~J}$ coupling constant between the $\mathrm{H}^{\prime}$ ' and $\mathrm{H}^{\prime}$ ' 
atoms $\left({ }^{3} \mathrm{~J}_{\mathrm{H} 1^{\prime}-\mathrm{H} 2^{\prime}}\right)(13,17)$, and the furanose ring pucker (Figure 5 , right). A lower value of ${ }^{3} \mathrm{~J}_{\mathrm{H} 1^{\prime}-\mathrm{H} 2^{\prime}}$ indicates a higher proportion of the 3 '-endo configuration. Our observations show that cytidine exhibits a higher degree of 3'-endo configuration relative to other nucleotides at the $\mathrm{N} 1$ position. We hypothesized that this higher fraction of 3'-endo conformation could potentially contribute to the faster reaction rate of imidazolium-bridged dinucleotides with $\mathrm{C}$ at the $\mathrm{N} 1$ position.

To further test the effect of the ribose sugar pucker of the substrate of primer extension reactions, we also investigated the sugar pucker of ribonucleotide monomers. We measured the values of ${ }^{3} \mathrm{~J}_{\mathrm{H} 1}{ }^{\prime}-\mathrm{H}{ }^{\prime}$ of the four 5'-monophosphate ribonucleotides, with and without 2-aminoimidazolium activation, in solution (Figure 5, left). The ${ }^{3} \mathrm{~J}_{\mathrm{H} 1}$-H2' values for NMPs has been reported previously with minor differences from this work.(18) Cytidine and uridine show stronger preferences for the C3'-endo conformational compared to guanosine and adenosine. To determine whether the 3 '-endo conformation leads to a higher reaction rate for activated monomers, we conducted primer extension reactions with only one nucleotide binding site on the template (Figure S3). Here, 2Al-activated ribonucleotide monomer $\left({ }^{*} \mathrm{~N}\right)$ is stacked with $\mathrm{G}$ at 3 '-end of primer and with another $G$ at the 5 '-end of the downstream helper oligonucleotide. Although the affinities of ${ }^{*} \mathrm{C}$ and ${ }^{*} \mathrm{U}$ are much lower than ${ }^{*} \mathrm{G}$ and ${ }^{*} \mathrm{~A}$ (as expected due to decreased staking energies), ${ }^{*} \mathrm{C}$ has higher $k_{\text {obs max }}$ compared to * $\mathrm{G}$, and quite surprisingly * $\mathrm{U}$ has higher $k_{\mathrm{obs} \max }$ compared to *A.

To further examine the effect of sugar pucker on reactivity and to control for any N1 or N2 nucleobaseidentity effects, we synthesized the imidazolium-bridged dinucleotides of four sugar-modified guanosines with different conformational preferences, and measured their performance in the primer extension reaction without the downstream helper (Figure 6). By using the same guanosine base in these modified dinucleotides, any difference in the primer extension behavior should be a result of the different 2' functional group on the sugar. The maximal rate of reaction correlates strongly with the ratio of the C3'endo to $\mathrm{C} 2$ '-endo conformers, as calculated from the values of ${ }^{3} \mathrm{JH}^{1}-\mathrm{H} 2^{\prime}$ (Figure $6 \mathrm{C}$ ). The fastest reacting imidazolium dimer is the locked-nucleic acid (LNA), which can only exist in the C3'-endo conformation; the slowest reacting dinucleotide is the DNA version, which prefers the C2'-endo conformation. Our results confirm that the primer extension reaction is faster when the imidazolium-bridged intermediate is pre-organized with the nucleotide sugars in the C3'-endo conformation.

\section{Potential solutions to the weak binding and poor reactivity of specific dinucleotide intermediates.}

We propose two strategies to overcome the poor efficiency of primer extension that results from the poor binding and reactivity of certain imidazolium-bridged dinucleotides. First, we suggest that monomers that are imidazolium-bridged to oligonucleotides will bind more strongly to the template, and may therefore enhance the rate of nonenzymatic primer extension. Our lab has previously demonstrated that an activated helper oligonucleotide catalyzes nonenzymatic primer extension with an activated monomer that is sandwiched between the primer and the helper oligonucleotide.(19) With the recent discovery of the imidazolium-bridged dinucleotide intermediate, it now seems likely that the catalytic effect of the activated downstream oligonucleotide is due to the formation of monomer-bridged-oligonucleotides. In order to test 
this hypothesis directly, we synthesized a series of monomer-bridged-oligonucleotides and measured their rates of nonenzymatic primer extension as a function of concentration (Figure 7). For this set of experiments, we used a primer with a 3'-terminal $C$ and no downstream helper oligonucleotide, to minimize the effect of stacking interactions on binding. Remarkably, compared to $A^{*} C, A^{*} C G$ is 7 -fold better in maximum nonenzymatic primer extension rate, over 20 -fold better in binding, and almost 150 fold better in $k_{\text {obs } \max } / \mathrm{K}_{\mathrm{m}}$. As the bridged oligo gets longer, the $k_{\mathrm{obs}}$ max reaches a plateau; this $k_{\text {obs } \max }$ plateau is comparable to the highest $k_{\text {obs max }}$ observed in the sandwich model with bridged dinucleotides. This finding suggests that the large differences observed between the affinities of different bridged dinucleotides can be reduced with the use of monomer-bridged-oligos. Moreover, since the affinity increases significantly as the intermediate gets longer, the maximum reaction rate is reached at much lower concentrations (Figure 7B).

As an alternative potential solution to the problems resulting from the wide range of affinities of the different bridged dinucleotides, we propose that the use of certain noncanonical nucleotides may lead to more uniform binding and thus more uniform rates of nonenzymatic primer extension. The stereoelectronic character of the nucleobase can tune the sugar pucker confirmation of a nucleotide,(20) and 2-thiolated pyrimidines (2-thioU, sU; 2-thio- $\mathrm{C}, \mathrm{sC}$ ) have been shown to have an increased preference for the C3'-endo configuration.(21) Furthermore, the sU:A base pair is stronger than U:A base pair,(22) while the SC:I (inosine) base pair(23) is weaker than the C:G base pair because it has 2 instead of 3 hydrogen bonds per base pair. To examine the conformations, affinities and rates of the corresponding imidazolium-bridged dinucleotides, we synthesized the 2-thio-uridine homodimer ( $\left.\mathrm{sU}^{*} \mathrm{sU}\right)$ and the 2-thiocytidine homodimer $\left(\mathrm{SC}^{*} \mathrm{SC}\right)$. The ${ }^{3} \mathrm{JH}^{\prime}{ }^{\prime}-\mathrm{H} 2^{\prime}$ value of $\mathrm{SU}^{*} \mathrm{SU}$ is around 2.3, lower than that of any other nucleotide reported in Figure 5. Surprisingly, $\mathrm{SC}^{*} \mathrm{SC}$ has a ${ }^{3} \mathrm{~J}_{\mathrm{H} 1^{\prime}-\mathrm{H} 2^{\prime}}$ around 0 , indicating an almost entirely C3'-endo configuration in solution. We then measured the nonenzymatic primer extension rate of the two homo-dimers in the sandwich model with sU:A and sC:I base pairs at the binding pocket (Figure 8). The $\mathrm{SU}^{*} \mathrm{sU}$ dinucleotide shows almost 8-fold increase and the $\mathrm{SC}^{*} \mathrm{sC}$ has almost 3-fold increase in the $k_{\text {obs max. }}$ Note that the $\mathrm{SC}^{*} \mathrm{sC}$ has an extremely high $k_{\text {obs } \max }=220 \mathrm{~h}^{-1}$, which means almost half of the primer can be extended in just 8 seconds. In addition, the $\mathrm{Km}$ of $\mathrm{sU}^{*} \mathrm{sU}$ has been improved by about 2-fold, while the binding of $\mathrm{SC}^{*} \mathrm{SC}$ on a $3^{\prime}-\mathrm{II}$ template is 20 -fold weaker compared to $\mathrm{C}^{*} \mathrm{C}$ on a 3'-GG template. (Figure S2, S6). As a result, the over 130-fold affinity difference between the two bridged dinucleotides has been decreased to only 3-fold. We suggest that the use of noncanonical nucleotides and monomer-bridgedoligonucleotides may allow for significantly increased rates of nonenzymatic primer extension and decreased differences between the affinities of different bridged dinucleotides for mixed sequence templates.

\section{DISCUSSION}

The recent discovery that the dominant pathway for nonenzymatic primer extension involves imidazoliumbridged dinucleotides as a covalent intermediate enabled us to reevaluate the kinetics of RNA template 
copying. Activated monomers first react with each other to form intermediate dimers, then bind to the template and react with the primer. Early studies of nonenzymatic RNA copying used activated mononucleotides, instead of purified intermediates; thus, the observed kinetics reflected the combination of distinct steps in the multi-step reaction. Nevertheless, these pioneering studies clearly showed that primer extension with $\mathrm{C}, \mathrm{G}$ and $\mathrm{A}$ is much more efficient than with $\mathrm{U}$.(8) Subsequent work showed that short activated helper oligonucleotides enable copying of mixed sequence templates, and facilitated the incorporation of $A$ and $U$ (19). Recent experiments using the purified dicytidine $C^{*} C$ intermediate provided for the first time a more accurate and comprehensive view of this multi-step chemical process.(5) Here, we have extended this analysis to a systematic study of the reaction kinetics of all ten canonical imidazolium-bridged dinucleotide intermediates. Our results show reasonably fast reaction rates $(\sim 6$ to $85 \mathrm{~h}^{-1}$ ) in all sixteen reactions when the intermediates are at saturating concentrations. However, the affinities of these intermediates for complementary templates exhibited a $>7,000$ fold range, from the very weak binding of $U^{*} U$ to the very tight binding of $G^{*} G$. Given that equimolar activated monomers equilibrate to give roughly equimolar concentrations of bridged intermediates, it is clear that previous efforts to copy mixed sequence templates failed due to the extremely sub-saturating concentrations of the most weakly binding intermediates $\left(U^{*} U, U^{*} A, A^{*} C\right.$ and $\left.U^{*} G\right)$. Additional experiments will be required to see if adjusting the concentrations of monomers leads to enhanced copying of mixed sequence templates.

Our study only compared the reaction rates of each intermediate when they are sandwiched between guanosines at the end of the primer and the beginning of the downstream helper oligonucleotide. When stacking against other nucleobases, the reaction rates and affinities of the intermediates are likely to differ. In particular, the weak stacking of intermediates with the 3 '-terminal $U$ of a primer, or of bridged intermediates with $\mathrm{a} U$ in the downstream position, could make it difficult to reach saturating concentrations of intermediate at specific template positions, and could thus contribute to the difficulty of copying arbitrary template sequences. Thus other ways of modulating the affinity of bridged intermediates for the template may be needed to enable efficient sequence-general copying.

Our studies show that the efficiency of nonenzymatic primer extension with imidazolium-bridged dinucleotide intermediates is determined in part by the affinity of the dinucleotide for the primer/template/helper complex, but also by the sugar pucker of the incoming nucleotide. The affinities of the dinucleotide intermediates are affected by both the Watson-Crick base pairing and by the stacking interactions with neighboring nucleotides. Interestingly, the highest affinity intermediates also show the fastest reaction rates, even at saturating concentrations. While the reasons for this phenomenon remain unknown, we suggest that a series of strong interactions may stabilize the optimal configuration of the bound dinucleotides for reaction with the primer. It is also possible that weak interactions with the template-adjacent nucleotide (e.g. with $U$ ) may allow that nucleotide to transiently become unpaired to the template, even though the dinucleotide as a whole remains bound. The effect of sugar pucker in the imidazolium-bridged dinucleotide was unexpected, but emerged from the finding that the superior 
reactivity of imidazolium-bridged dinucleotides in which $\mathrm{N} 1$ was $\mathrm{C}$ correlated with the stronger preference of $C$ for the $C 3$ '-endo configuration, both as a monomer and in the intermediate. The preference of $C$ for the C3'-endo configuration has been previously reported. $(20,24)$ By measuring the rate of primer extension with $G^{*} G$ analogs with different sugar 2'-substituents, we confirmed that the $C 3$ '-conformation of the dinucleotide intermediate contributes to a faster rate of primer extension. The reason for this effect is not immediately obvious, since the sugars of the N1 and N2 nucleotides are remote from the site of the reaction. However, we suggest that the conformation of the sugar of the N1 nucleotide may influence the position of the reactive phosphate, relative the primer 3'-hydroxyl.

The wide range of observed reaction rates and affinities among the ten different imidazolium-bridged dinucleotide intermediates clearly contributes to the difficulty of copying mixed sequence RNA templates. We asked whether this problem could potentially be overcome by employing either (i) noncanonical nucleotides, or (ii) activated short helper oligonucleotides. These potential solutions have been proposed before, $(19,25)$ but without recognition of the role of bridged-intermediates. Thiolated pyrimidine nucleotides have a strong preference for the $\mathrm{C}^{\prime}$ '-endo sugar conformation, and our experiments show that the $\mathrm{SU}^{*} \mathrm{SU}$ and $\mathrm{SC}^{*} \mathrm{~s} \mathrm{C}$ bridged dinucleotides exhibit significantly faster reaction rates than the canonical $U^{*} U$ and $C^{*} C$ intermediates. This is of particular interest given that both $\mathrm{sC}$ and $\mathrm{sU}$ can be generated form the prebiotic Sutherland pathway to the pyrimidine ribonucleotides.(26) As an alternative approach, we also prepared a series of monomer-bridged-oligonucleotides and compared their primer extension reaction rates with that of a standard bridged dinucleotide. Monomer bridged to a trimer exhibited a template affinity three orders of magnitude stronger than that of a simple bridged dinucleotide. In a prebiotic RNA replication scenario in which many short oligonucleotides were present (as in the virtual circular genome model)(27), and could become activated, such monomer-bridged oligonucleotide species would form naturally, and may have played a key role in enabling nonenzymatic RNA replication. We are currently examining the efficiency and fidelity of nonenzymatic primer extension in the presence of both noncanonical nucleotides and monomer-bridged-oligonucleotide species. We hope that these studies will lead to prebiotically realistic scenarios under which efficient nonenzymatic replication may take place.

\section{SUPPLEMENTARY DATA}

Supplementary data are available at NAR online.

\section{ACKNOWLEDGEMENTS}

We thank Dr. Marco Todisco, Dr. Li Li, Dr. Daniel Duzdevich, Dr. Seohyun Chris Kim and Lydia Pazienza for helpful discussion and technical assistance. We also thank all members of the Szostak laboratory for helpful feedback.

\section{FUNDING}


National Science Foundation [CHE-1607034 to J.W.S.]; Simons Foundation [290363 to J.W.S.]. J.W.S. is an investigator of the Howard Hughes Medical Institute.

\section{CONFLICT OF INTEREST}

The authors declare no conflicts of interest.

\section{REFERENCES}

1. Weimann,B.J., Lohrmann,R., Orgel,L.E., Schneider-Bernloehr,H. and Sulston,J.E. (1968) Templatedirected synthesis with adenosine-5'-phosphorimidazolide. Science, 161, 387.

2. Inoue,T. and Orgel,L.E. (1982) Oligomerization of (guanosine 5'-phosphor)-2-methylimidazolide on poly(C): An RNA polymerase model. J. Mol. Biol., 162, 201-217.

3. Wu,T. and Orgel,L.E. (1992) Nonenzymatic template-directed synthesis on hairpin oligonucleotides. 3. Incorporation of adenosine and uridine residues. J. Am. Chem. Soc., 114, 7963-7969.

4. Walton,T. and Szostak,J.W. (2016) A Highly Reactive Imidazolium-Bridged Dinucleotide Intermediate in Nonenzymatic RNA Primer Extension. J. Am. Chem. Soc., 138, 11996-12002.

5. Walton,T. and Szostak,J.W. (2017) A Kinetic Model of Nonenzymatic RNA Polymerization by Cytidine5'-phosphoro-2-aminoimidazolide. Biochemistry, 56, 5739-5747.

6. Duzdevich,D., Carr,C.E., Ding,D., Zhang,S.J., Walton,T.S. and Szostak,J.W. (2021) Competition between bridged dinucleotides and activated mononucleotides determines the error frequency of nonenzymatic RNA primer extension. Nucleic Acids Res., 49, 3681-3691.

7. Duzdevich,D., Carr,C.E. and Szostak,J.W. (2020) Deep sequencing of non-enzymatic RNA primer extension. Nucleic Acids Res., 48, e70.

8. Li,L., Prywes,N., Tam,C.P., Oflaherty,D.K., Lelyveld,V.S., Izgu,E.C., Pal,A. and Szostak,J.W. (2017) Enhanced nonenzymatic RNA copying with 2-aminoimidazole activated nucleotides. J. Am. Chem. Soc., 139, 1810-1813.

9. Fahrenbach,A.C., Giurgiu,C., Tam,C.P., Li,L., Hongo,Y., Aono,M. and Szostak,J.W. (2017) Common and Potentially Prebiotic Origin for Precursors of Nucleotide Synthesis and Activation. J. Am. Chem. Soc., 139, 8780-8783.

10. Walton,T. and Szostak,J.W. (2017) A Kinetic Model of Nonenzymatic RNA Polymerization by Cytidine-5'-phosphoro-2-aminoimidazolide. Biochemistry, 56, 5739-5747.

11. Malek-Adamian,E., Patrascu,M.B., Jana,S.K., Martínez-Montero,S., Moitessier,N. and Damha,M.J. (2018) Adjusting the Structure of 2'-Modified Nucleosides and Oligonucleotides via C4'- $\alpha-F$ or C4'- $\alpha-$ OMe Substitution: Synthesis and Conformational Analysis. J. Org. Chem., 83, 9839-9849.

12. Norberg,J. and Nilsson,L. (1995) Stacking Free Energy Profiles for All 16 Natural Ribodinucleoside Monophosphates in Aqueous Solution. J. Am. Chem. Soc., 117, 10832-10840.

13. Altona,C. and Sundaralingam,M. (1973) Conformational analysis of the sugar ring in nucleosides and 
nucleotides. Improved method for the interpretation of proton magnetic resonance coupling constants. J. Am. Chem. Soc., 95, 2333-2344.

14. Kozlov,I.A., Politis,P.K., Van Aerschot,A., Busson,R., Herdewijn,P. and Orgel,L.E. (1999) Nonenzymatic Synthesis of RNA and DNA Oligomers on Hexitol Nucleic Acid Templates: The Importance of the A Structure. J. Am. Chem. Soc., 121, 2653-2656.

15. Zhang,W., Walton,T., Li,L. and Szostak,J.W. (2018) Crystallographic observation of nonenzymatic RNA primer extension. Elife, 7, e36422.

16. Giurgiu,C., Fang,Z., Aitken,H.R.M., Kim,S.C., Pazienza,L., Mittal,S. and Szostak,J.W. (2021)

Structure-Activity Relationships in Nonenzymatic Template-Directed RNA Synthesis. Angew. Chemie, 10.1002/ange.202109714.

17. Zhang,N., Zhang,S. and Szostak,J.W. (2012) Activated Ribonucleotides Undergo a Sugar Pucker Switch upon Binding to a Single-Stranded RNA Template. J. Am. Chem. Soc, 134, 3691-3694.

18. Ts'o,P.O.P., Kondo,N.S., Schweizer,M.P. and Hollis,D.P. (1969) Studies of the Conformation and Interaction in Dinucleoside Mono- and Diphosphates By Proton Magnetic Resonance. Biochemistry, 8, 997-1029.

19. Prywes,N., Blain,J.C., Del Frate,F. and Szostak,J.W. (2016) Nonenzymatic copying of RNA templates containing all four letters is catalyzed by activated oligonucleotides. Elife, 5, e17756.

20. Plavec,J., Thibaudeau,C. and Chattopadhyaya,J. (1996) How do the energetics of the stereoelectronic gauche and anomeric effects modulate the conformation of nucleos(t)ides? Pure Appl. Chem., 68, 2137-2144.

21. Kawai,G., Yamamoto,Y., Watanabe,T., Yokoyama,S., Kamimura,T., Masegi,T., Sekine,M., Hata,T., limori,T. and Miyazawa,T. (1992) Conformational Rigidity of Specific Pyrimidine Residues in tRNA Arises from Posttranscriptional Modifications That Enhance Steric Interaction between the Base and the 2'-Hydroxyl Group. Biochemistry, 31, 1040-1046.

22. Larsen,A.T., Fahrenbach,A.C., Sheng,J., Pian,J. and Szostak,J.W. (2015) Thermodynamic insights into 2-thiouridine-enhanced RNA hybridization. Nucleic Acids Res., 43, 7675-7687.

23. Ohkubo,A., Nishino,Y., Ito,Y., Tsunoda,H., Seio,K. and Sekine,M. (2012) Formation of new base pairs between inosine and 5-methyl-2-thiocytidine derivatives. Org. Biomol. Chem., 10, 2008-2010.

24. Hocquet,A., Leulliot,N. and Ghomi,M. (2000) Ground-State Properties of Nucleic Acid Constituents Studied by Density Functional Calculations. 3. Role of Sugar Puckering and Base Orientation on the Energetics and Geometry of 2'-Deoxyribonucleosides and Ribonucleosides. J. Phys. Chem. B, 104, 4560-4568.

25. Heuberger,B.D., Pal,A., Del Frate,F., Topkar,V. V. and Szostak,J.W. (2015) Replacing uridine with 2thiouridine enhances the rate and fidelity of nonenzymatic RNA primer extension. J. Am. Chem. Soc., 137, 2769-2775.

26. Xu,J., Tsanakopoulou,M., Magnani,C.J., Szabla,R., Šponer,J.E., Šponer,J., Góra,R.W. and Sutherland,J.D. (2017) A prebiotically plausible synthesis of pyrimidine $\beta$-ribonucleosides and their 
bioRxiv preprint doi: https://doi.org/10.1101/2021.10.06.463393; this version posted October 6, 2021. The copyright holder for this preprint

(which was not certified by peer review) is the author/funder, who has granted bioRxiv a license to display the preprint in perpetuity. It is made available under aCC-BY 4.0 International license.

phosphate derivatives involving photoanomerization. Nat. Chem., 9, 303-309.

27. Zhou,L., Ding,D. and Szostak,J.W. (2021) The virtual circular genome model for primordial RNA replication. $R N A, 27,1-11$. 

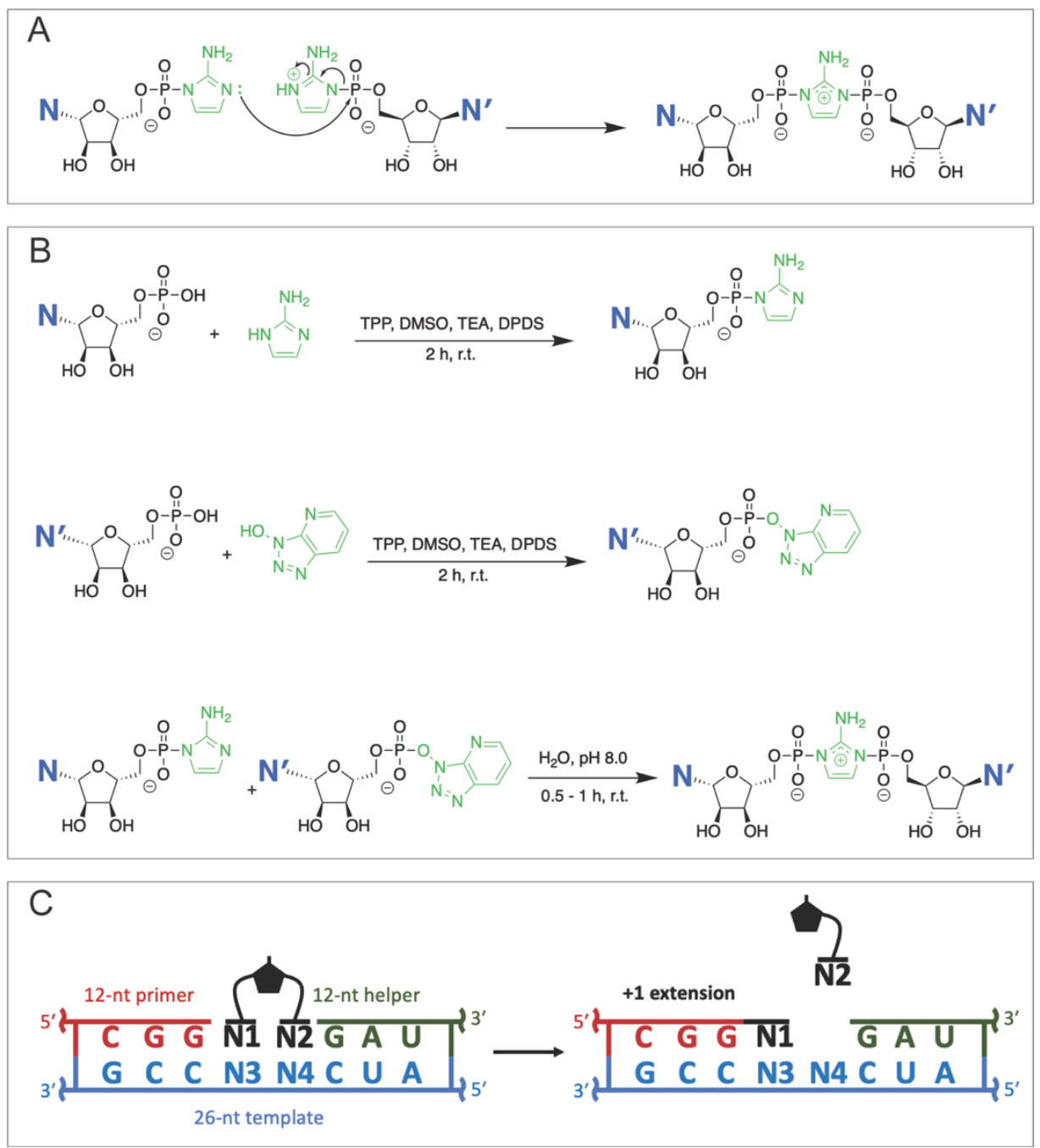

Figure 1: (A) The spontaneous formation of 2-aminoimidazolium-bridged dinucleotides in solution. (B) Scheme for chemical synthesis of imidazolium-bridged dinucleotides. (C) Scheme for primer extension experiments. Primer extension reactions were carried out with $1.5 \mu \mathrm{M}$ primer, $2.5 \mu \mathrm{M}$ template, $3.5 \mu \mathrm{M}$ helper, $100 \mathrm{mM} \mathrm{MgCl}_{2}$ and $200 \mathrm{mM}$ Tris $\mathrm{pH} 8.0$. 


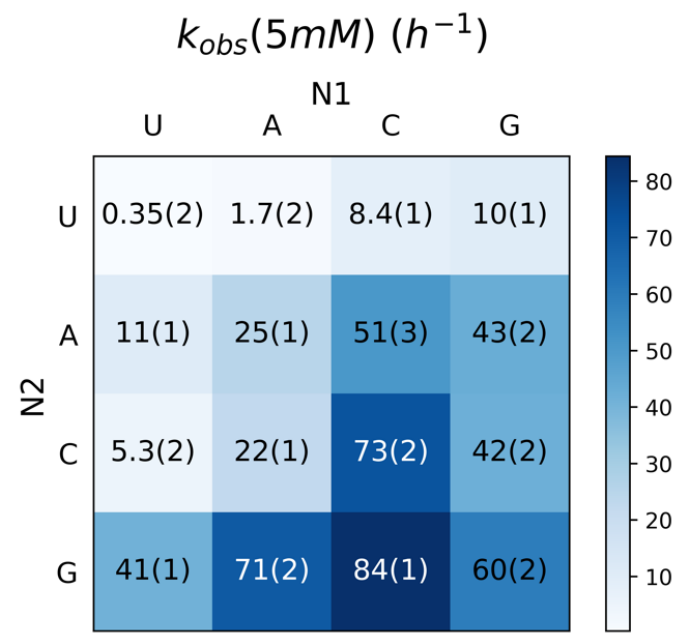

Figure 2: $k_{\mathrm{obs}}$ of nonenzymatic primer extension reactions of imidazolium-bridged dinucleotide intermediates $\mathrm{N} 1{ }^{*} \mathrm{~N} 2$ at $5 \mathrm{mM}$. Note that intermediates $\mathrm{N} 1{ }^{*} \mathrm{~N} 2$ and $\mathrm{N} 2{ }^{*} \mathrm{~N} 1$ are identical, but are bound to templates with reverse orientation e.g. 5'-N3-N4-3' and 5'-N4-N3-3'. The experimental design is described in Fig $1 \mathrm{C}$.

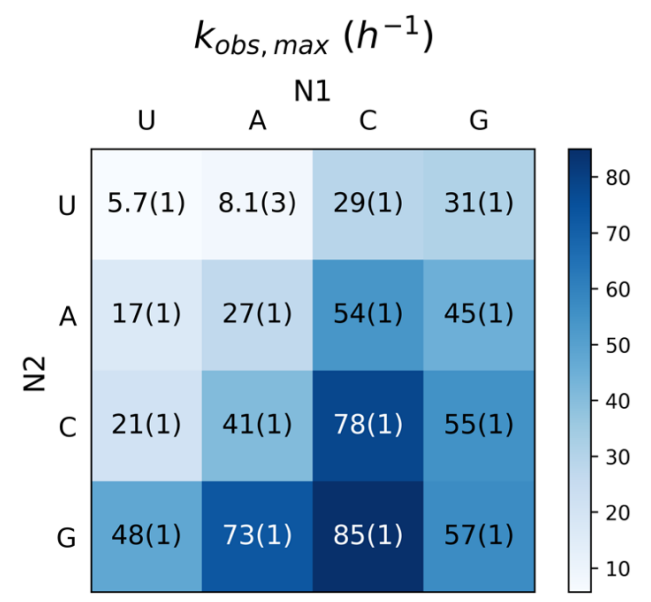

Figure 3: $k_{\text {obs max }}$ of nonenzymatic primer extension reactions from 16 Watson-Crick base pairing of dinucleotide intermediates $\mathrm{N} 1{ }^{*} \mathrm{~N} 2$. The experimental design is as described in Fig $1 \mathrm{C}$. 


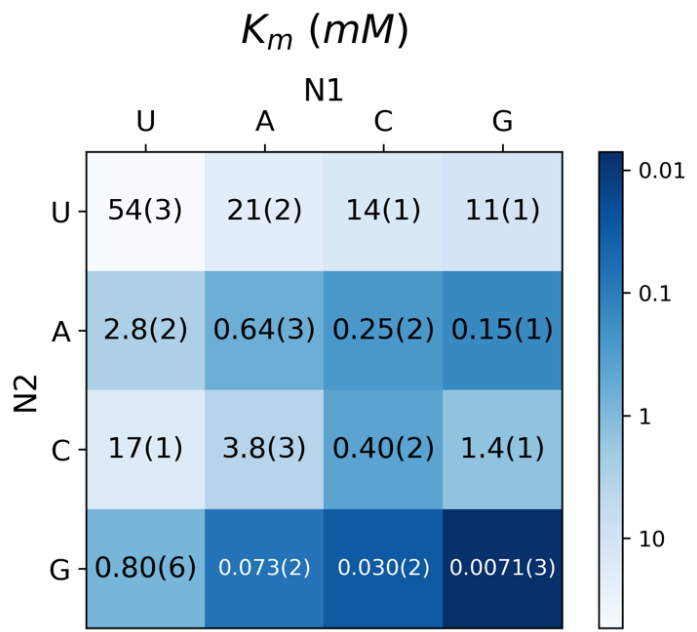

Figure 4: $K_{m}$ of imidazolium-bridged dinucleotide intermediates in nonenzymatic primer extension reactions, for all 16 possible Watson-Crick N1*N2/template combinations. The experimental design is as described in Fig $1 \mathrm{C}$.
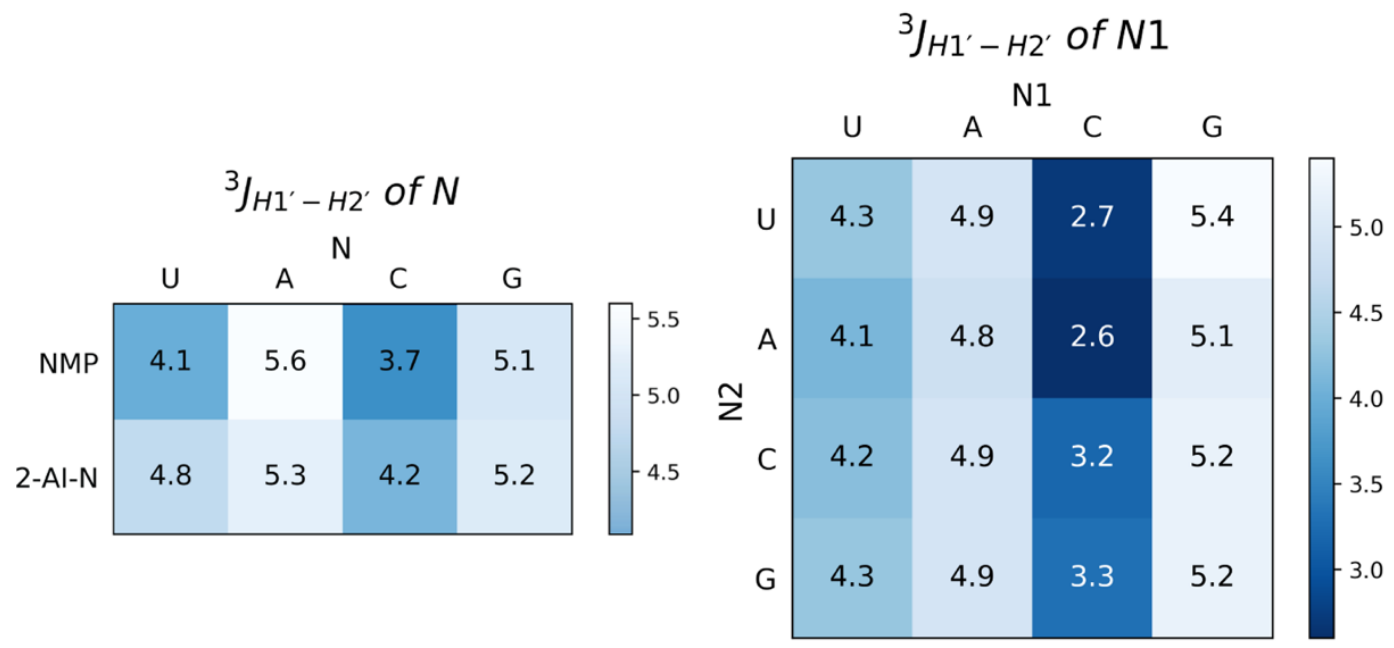

Figure 5: Left: ${ }^{3} \mathrm{~J}_{\mathrm{H} 1}{ }^{-}-\mathrm{H}{ }^{\prime}$ of the nucleotide in monophosphate form and in the activated monomer form measured at $\mathrm{pH}$ 8.0. Right: ${ }^{3} \mathrm{~J}_{\mathrm{H} 1}{ }^{\prime}-\mathrm{H}{ }^{\prime}$ of $\mathrm{N}_{1}$ nucleotide in the intermediate $\mathrm{N}_{1}{ }^{*} \mathrm{~N}_{2}$ measured at $\mathrm{pH}$ 8.0. A lower value of ${ }^{3} \mathrm{JH}^{1}$-H2' represents a higher degree of 3 '-endo configuration. 

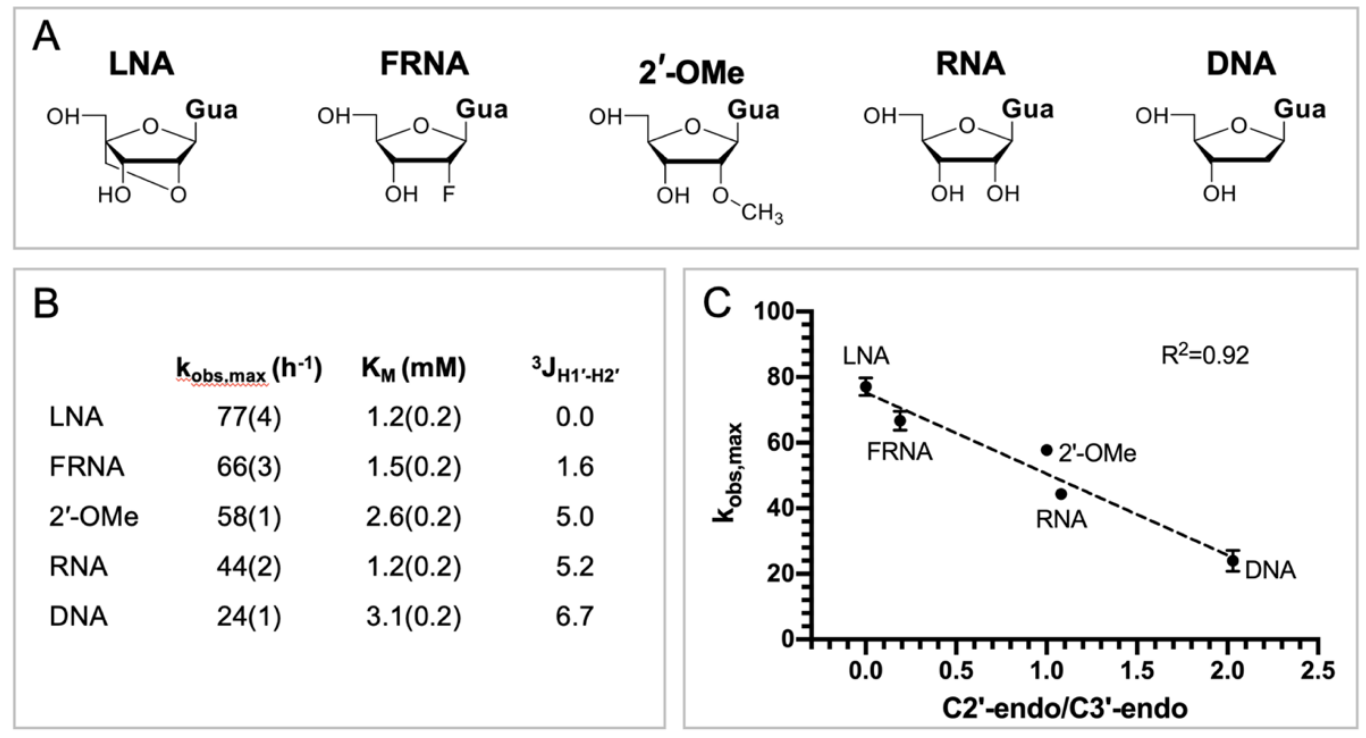

Figure 6: Primer extension with 2' modified di-guanosine imidazolium-bridged dinucleotides. (A) Chemical structures of different modified guanosine nucleotides used to form the dinucleotide intermediates. (B) Kinetic parameters and ${ }^{3} \mathrm{~J}_{\mathrm{H} 1}$-H2' for each modified di-guanosine imidazolium-bridged dinucleotide $(C)$ The correlation between the maximal rate of the reaction and the ratio of the two conformers. 

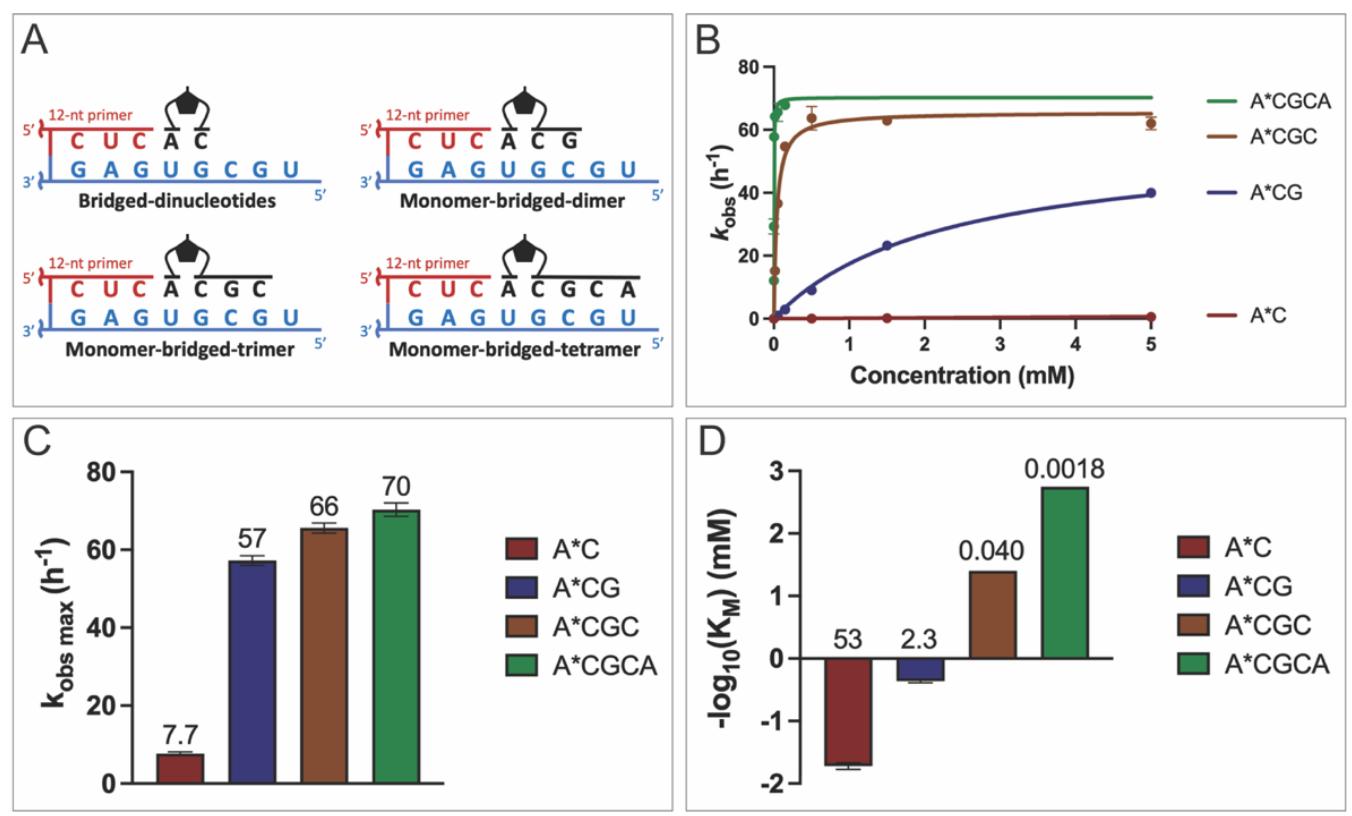

Figure 7: Primer extension with monomers imidazolium-bridged to oligonucleotides. The primer ends in a 3'-terminal C; there is no downstream helper. (A) Schematic representation of the nonenzymatic primer extension setup. (B) Michaelis-Menten curves plotted for 0-5 $\mathrm{mM}$ of the monomer-bridged-oligonucleotide intermediates. See the SI for the Michaelis-Menten plots of each individual monomer-bridged oligonucleotide. The comparison of $(C) k_{\text {obs max }}$ and $(D)-\log _{10}\left(K_{M}\right)$ between $A^{*} C, A^{*} C G, A^{*} C G C, A^{*} C G C A$ are shown. The values of $k_{0 b s} \max$ and $\mathrm{K}_{\mathrm{M}}$ are indicated above each bar. 


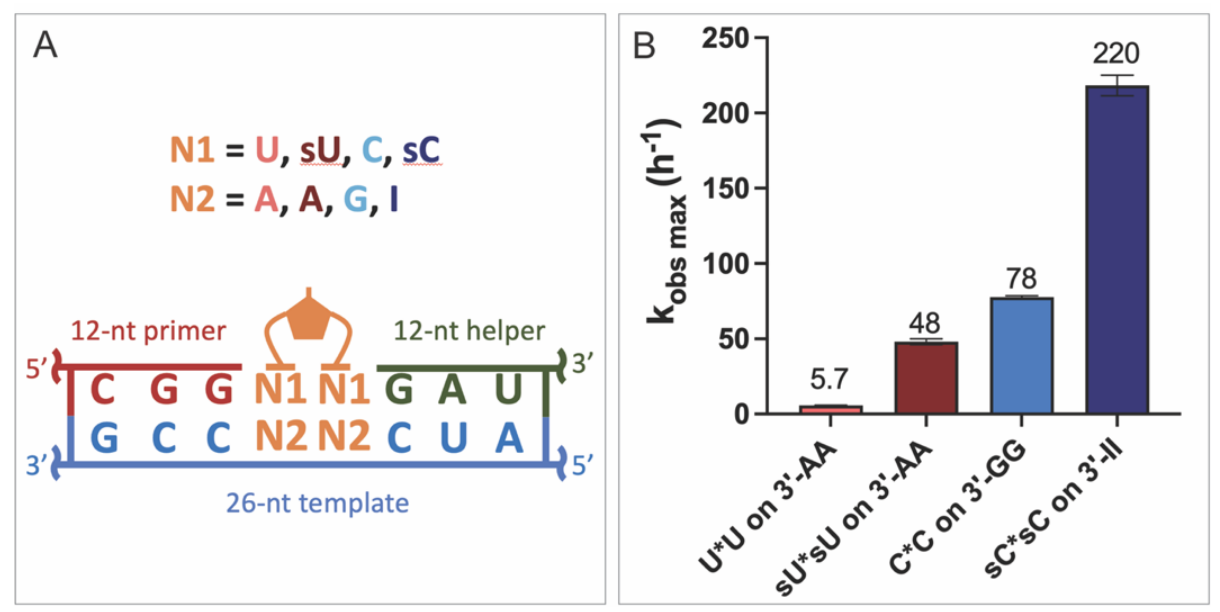

Figure 8: Primer extension with noncanonical bridged dinucleotides. (A) Scheme for the experiments.

The template for $\mathrm{SU}^{*} \mathrm{sU}$ had two adenosines while the template for $\mathrm{SC}^{*} \mathrm{sC}$ had two inosines in the binding pocket. (B) Comparison of $k_{\text {obs max }}$ between $U^{*} U, s U^{*} s U, C^{*} C, s C^{*} s C$ in the sandwich model. For detailed description please see the SI. 